Tohoku J. exp. Med., 1968, 95, 15-33

\title{
Histometrical Studies on the Kidneys in Diabetes Mellitus
}

\author{
Norio Fukuhara \\ The Third Department of Internal Medicine (Prof. S. Yamagata) and \\ the First Department of Pathology (Prof. N. Suwa), \\ Tohoku University School of Medicine, Sendai
}

Though the histological glomerular lesions in diabetes mellitus appear in two distinet types, the diffase and nodular, the former lacks pathognomonic features, and its differentiation from mild chronic glomerulonephritis has been practically impossible. A histometrical method was introduced into the present study to establish a diagnostic basis of slight diabetic nephropathy. The relative reduction of mesangial nuclei per unit area of mesangial tissue was characteristic of diabetic glomerular lesions and was useful for separating diabetic from mild chronic glomerulonephritic lesions. This was due to hyaline thickening of the mesangial tissue in diabetics without nuclear proliferation.

Diabetic glomerular lesions were further quantitatively evaluated and correlated to a number of clinical symptoms. Positive correlation was confirmed between the grade of glomerular lesions and serum lipid levels. The correlation was regarded as of pathogenetic significance in the diffuse glomerular lesion.

The development of coarse nodular glomerular lesions was ascribed to mesangiolytic changes with subsequent plasmal infiltration into the mesangia! tissue and capillary thrombosis. Locally accentuated hyaline deposits in the mesangial tissue occasionally simulated small nodular lesions, but they did not grow to typical large nodules without preceding mesangiolytic changes.

Since Kimmelstiel and Wilson ${ }^{1}$ reported first a striking hyaline thickening of the intercapillary connective tissue of the glomerulus, the lesion has been generally accepted as being pathognomonic of diabetes mellitus. However, the coarse nodular thickening consisting of hyaline mass has a fairly large diameter and does not seem to develop to its complete form in a short period. There might be a precursory lesion of milder degree. Bell ${ }^{2}$ stated on his exact observations that the glomerular lesions in diabetes could be divided into two distinct types, the nodular and diffuse. He added in his monograph that diabetic glomerular changes in their early stages consisted in intercapillary deposit of hyaline mass, which later developed to the nodular lesions.

Although there was every evidence that these diffuse lesions were induced by diabetes, they were devoid of pathognomonic histological features and could be scarcely discriminated from mild glomerular lesions in chronic glomerulonephritis. ${ }^{3.4}$ When the diffuse lesions are very slight, it becomes utterly impossible to recognize their diabetic origin. Bell ${ }^{5}$ stated on this problem that

Received for publication, January 8, 1968. 
the diffuse glomerular lesions of the diabetic kidney were easily identified if they were accompanied by hyalinosis of the arterioles. However, the arteriolar hyalinization is not only an inconstant finding in slight diabetes, but also it may be obsarved in other diseases, and the difficulty doas not seem to be essentially overcome.

In the original dessription of Kimmelstiel and Wilson, capillary nuclei appeared well preserved in the initial stage of intercapillary hyaline deposition, but were destined to be embedded in dense hyaline mass, so that tho origin of the nuclei could not be ascertained. Because hyaline deposition was not accompanied with nuclear inc: zase, they ragarded this process as of degenerutive charaster. Until today this opinion has been supported by many investigators. ${ }^{6-9}$

If nuclear paucity is characteristic of diabetic nodular glomerular lesions, the same is reasonably expected in diffuse lesions in contrast with glomerulonephritis. However, the difference is not always convincing in routine histological examinations. Consequently, a histometrical method is introduced into the study in order to evaluate exactly the nuclear number in the glomeruli of diabetic nephropathy of diffuse type and mild chronic glomerulonephritis. If the difference is confirmed, the result would be not only of diagnostic value, but also effective in correlating clinical and pathological findings. In all the previous reports about clinicopathological corralation, ${ }^{4,10-12}$ the glomerular lesions were classified into the diffuse and nodular types and were submitted to separate treatments. However, since diabetic glomeralar lesions ar 3 supposed to progress from the diffuse type to the nodular, there is no reason to treat them independently, and it is appropriate to regard the difference of the two types as quantitative rather than qualitative and treat them on a common basis.

In the present study, an attempt was made to express the intensity of diabetic glomerular lesions with an index. A certain scora was given to each glomerulus on histological slides according to the grade of its pathological finding, as will be explained later. The difference of the diffuse and nodular types was thus rendered to a quantitative one. Although it is generally accepted that nodular lesions represent more advanced and severer glomerular involvement than diffuse ones, they are by no means a simple losal intensification of diffuse changes. The histogenesis of nodular lesions will be discussed based on the histological findings of biopsy specimens.

\section{Materials and Methods}

For the present study, biopsy specimens from 144 diabetics and from 24 patients with chronic glomerulonephritis were submitted to histological and histometrical examinations. Besides, the kidneys of 17 autopsy cases of diabetes mellitus and of 39 non-diabetic autopsy cases without noticeable renal involvement were used for the same purposes. All the tissue specimens were fixed in acidified Zenker-formalin and embedded in paraffin. Histological sections of $3 \mu$ thickness were stained with hematoxylin-eosin, periodic acid Schiff, Goldner's trichrome and 
Mallory's azan. Moreover, Sudan black B and methenamine silver stain were used when necessary.

For the quantitative evaluation of diabetic glomerular changes in reference to clinical symptoms, each glomerulus was scored according to the standards of Table 1 and Fig. 1. As the scores of the glomeruli in each case exhibited usually only limited scatter, the arithmetic mean of the score could be used as representing the grade of glomerular involvement in individual cases and designated as IDGL, index of diabetic glomerular lesions. In biopsy specimens all the glomeruli appearing on the histological sections were scored, while about 50 glomeruli were randomly taken in autopsy cases. Biopsy specimens with less than ten glomeruli were excluded from the study.

TABLE 1. Quantitative evaluation of glomerular lesions in diabetic kidneys

\begin{tabular}{|c|c|}
\hline Score & Histological findings of the glomerulus \\
\hline 0 & Normal. \\
\hline 1.0 & $\begin{array}{l}\text { Slight fibrous or hyaline thickening of the mesangium. The capillary } \\
\text { structure is perfectly preserved. The glomeruli of this grade cannot be } \\
\text { distinguished from those in mild chronic glomerulonephritis by means of } \\
\text { routine histological examination. }\end{array}$ \\
\hline 2.0 & $\begin{array}{l}\text { Diffuse hyaline thickening of mesangium without nodule formation. Distinct } \\
\text { hyaline mesangial thickening and nuclear paucity make the diagnosis of } \\
\text { diabetic glomerulosclerosis possible. }\end{array}$ \\
\hline 2.5 & $\begin{array}{l}\text { Locally accentuated hyaline deposits in the mesangium simulate small } \\
\text { nodules. Capillary structures are still essentially maintained. }\end{array}$ \\
\hline 3.0 & Large sharply demarcated hyaline nodules. \\
\hline 4.0 & Capillary thrombasis. The basic changes are those of grade 2 or 3 . \\
\hline
\end{tabular}

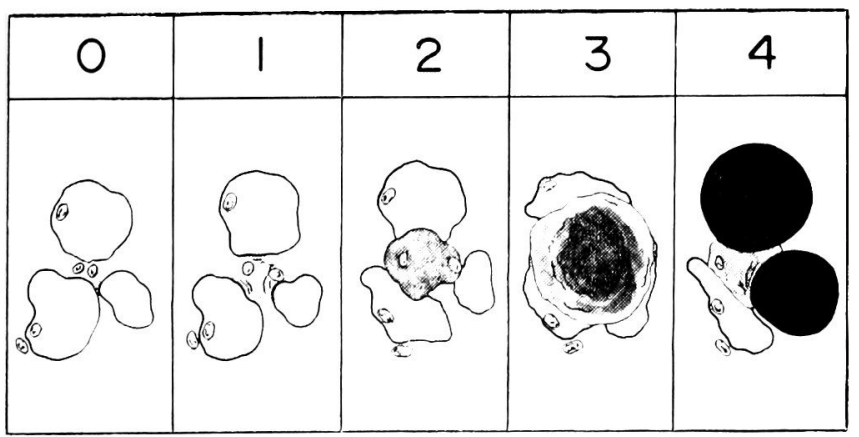

Fig. 1. The diabetic glomerular lesions and their scores are illustrated. For details see Table 1 . 
The IDGLs of the biopsy specimens were evaluated in relation to the age of the patients, duration of the disease, urinary sugar and protein contents, blood sugar level, total serum cholesterol level (measured by Zak-Henly's method), neutral fat (by van Handel-Zilversmit's method), total lipids, non-esterified fatty acids (by Dole's method), thromboplastic activity (by Biggs's method), endogenous creatinine clearance, para-aminohippuric acid clearance, serum non-protein nitrogen, blood pressure and diabetic retinopathy (Wagener). The autopsy cases were divided into three groups according to the direct cause of death and correlated to glomerular involvement.

Thirty-nine biopsy specimens of diabetic kidneys were selected for the histometrical treatment. The glomerular lesions in all the selected cases consisted in slight diffuse thickening of mesangial tissues and it was impossible to discriminate them with certainty from mild chronic glomerulonephritic changes by means of routine histological examinations.

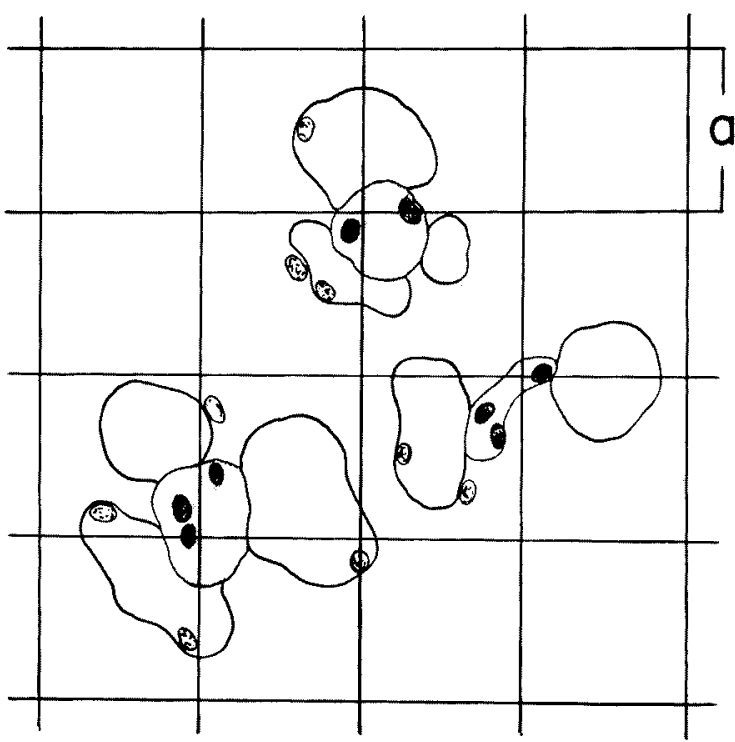

Fig. 2. A latticed eye-piece is used in the histometrical determination of the area occupied by the mesangial tissue on a glomerular section. For details see text.

The whole capillary tuft of a glomerular section was covered by a latticed ocular micrometer as shown in Fig. 2, and the number of the cross-points of the lattice falling on the mesangial tissue was counted. The mean number $n$ was thus obtained after a sufficiently large number of trials, while the lattice was overlaid randomly on the same glomerular section. When the interval of the lattice is $a$, the surface area $S$ of the mesangial tissues of the glomerulus on the histological section is given by:

$$
S=a^{2} n
$$


On the other hand, the number of mesangial nuclei $N$ was counted on the glomerular section. When $N$ is divided by $S$, the result gives the number of mesangial nuclei per unit area of the respective glomerulus. The procedure was repeated on a number of the glomeruli and the mean $N / S$ was used as the estimate representing the ratio of mesangial nuclei to mesangial volume.

\section{Results and Comments}

1) The number of mesangial nuclei per unit area of mesangial tissue on histological sections

When the number of mesangial nuclei per unit area of mesangial tissues of apparently normal kidneys was plotted against the age of the subjects on a doublelogarithmic coordinate system, a linear regression was observed with the equation of $Y=-0.0621 X+0.3947$, where $X$ and $Y$ were the logarithms of age and nuclear number respectively. The result is presented in Fig. 3 together with the zone made by rejection limits at $5 \%$ level.

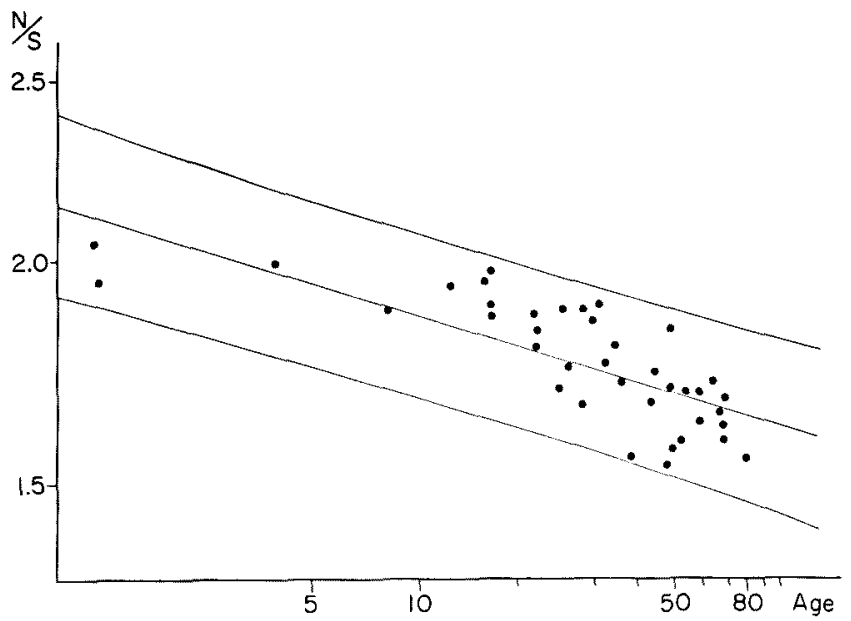

Fig. 3. The number of mesangial nuclei on a unit surface area of the mesangial section $N / S$ is presented in reference to age on a double-logarithmic coordinate system. The regression line together with a zone made by rejection limits is given in the diagram. Note gradual decrease of $N / S$ with advancing ages.

Because the regression coefficient is negative, the equation indicates a progressive reduction in the relative nuclear number with advancing ages even in apparently normal kidneys. This is due to hyaline thickening of the mesangial tissue in physiological aging without corresponding increase in the nuclear number. The mesangial tissue is seemingly the most sensitive portion in the glomerulus and is subject to repeated deposition of plasmal elements even under physiological conditions. 
The nuclear number per unit area of mesangial tissues was generally lower even in diabetic kidneys with slight glomerular involvement (Fig. 4). In about a half of the examined cases the estimates were still within the rejection limits of the normal kidney, while the other half gave the values below the lower rejection limit. In the latter cases relative reduction of mesangial nuclei in diabetic kidneys could be concluded. This is in agreement with the general histological feature of diffuse diabetic glomerular lesions characterized by hyaline thickening of the mesangial tissue without noticeable proliferation of the nuclei. The lesions of the examined cases were histologically so slight that diabetic glomerulosclerosis of diffuse type could hardly be diagnosed with certainty. Nevertheless, the histometrical treatment could reveal a relative reduction of mesangial nuclei or increased mesangial volume without corresponding nuclear proliferation.

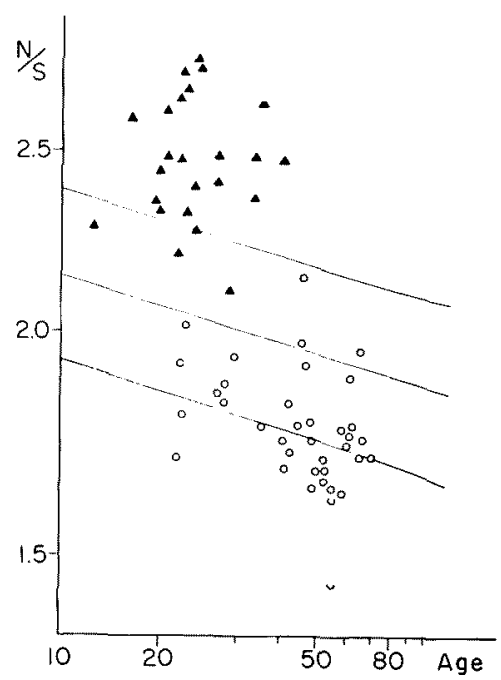

Fig. 4. The values of $N / S$ determined on biopsy specimens of diabetic nephropathy and chronic glomerulonephritis are presented in reference to the mean and rejection limits of the normal values. Diabetic nephropathy. * Chronic glomerulonephritis. Note the distinct distribution of the two groups.

On the contrary, the mesangial nuclear number exceeded the upper rejection limit of the normal kidney in the majority of the examined cases of chronic glomerulonephritis, although routine histological examinations could demonstrate only slight mesangial thickening without distinct proliferative process. The result indicates that nuclear proliferation predominates over the accompanying increase in mesangial volume even in mild glomerulonephritis. The difference in the behavior of the mesangial nuclei was thus found of diagnostic value in mild chronic glomerulonephritis and diabetic nephropathy.

The histometrically confirmed difference in the glomerular lesions between diabetes and chronic glomerulonephritis is also attributed to increased mesangial 
tissue without nuclear proliferation in diabetes. The result presents the evidence for plasmal deposition in the mesangial tissue in the course of diabetic glomerular changes. The exaggerated hyaline thickening of the tissue after plasmal infiltration in diabetes is probably associated with high lipid contents of the plasma in this disease. The same tendency is observed in pronounced hyaline thickening of the arterial intima, which contributes to accentuated arteriosclerosis of diabetics. The correlation of diabetic glomerular changes with plasmal lipids will be presented later.

\section{2) Clinicopathological correlation by means of IDGL}

a) Age: Low IDGLs were almost uniformly distributed in all the age groups especially when the indices were lower than 1.0. However, IDGLs higher than 2.2 were found only in the age group over 40 years (Fig. 5).

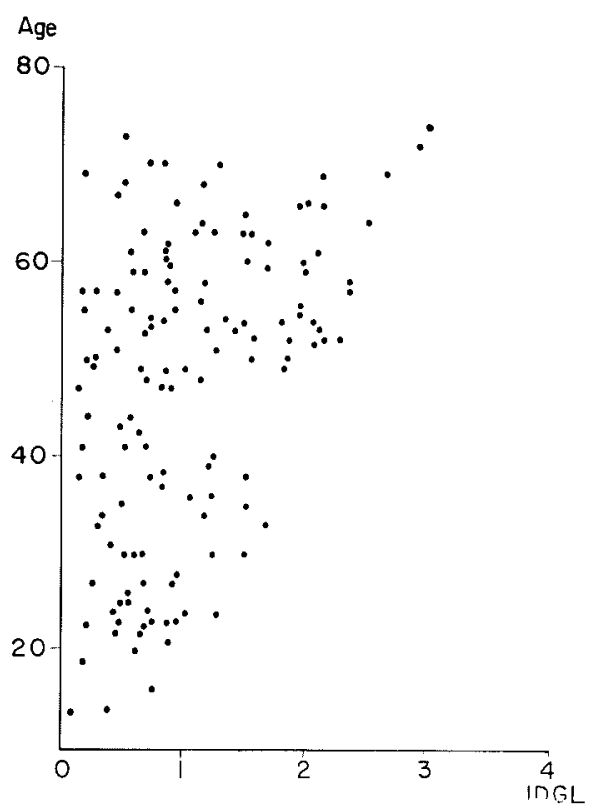

Fig. 5. IDGL against age. Note that high IDGLs are found only in advanced ages, while slight lesions are distributed uniformly over all ages.

b) Duration of diabetes: As the onset of diabetes is usually not exactly to date, the duration of the disease was estimated with the time since the establishment of the diagnosis. Low IDGLs were found even in cases of long duration of the disease and exhibited no definite correlation with the duration of the disease. On the contrary, severe lesions were observed only in cases of more than 7 years' duration (Fig. 6). The result, together with that of age, indicates that severe diabetic glomerular lesions can develop only after a considerably long duration of diabetic condition. 


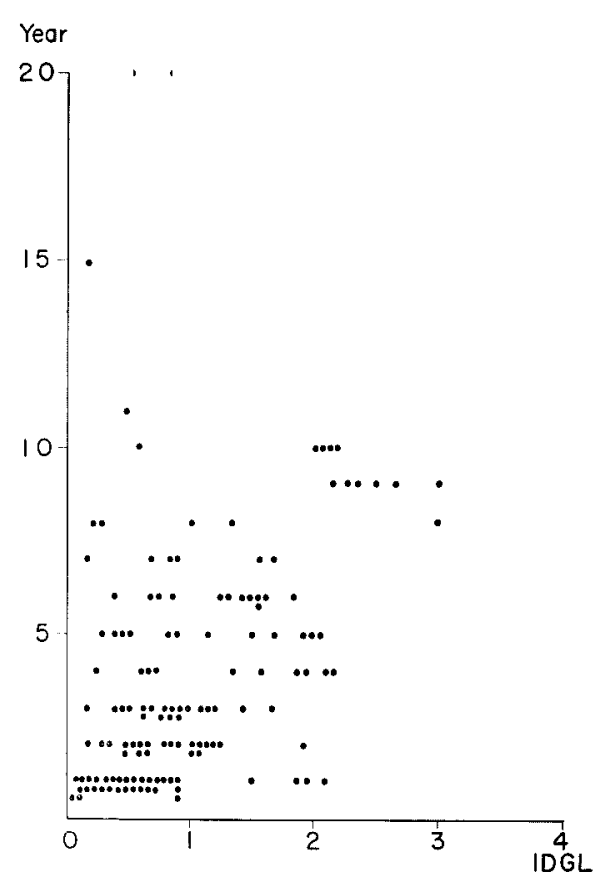

Fig. 6. IDGL against the duration after the established diagnosis. The same tendency is observed as in Fig. 5.

c) Albuminuria: Distinct albuminuria was found in 59 out of 132 cases. Severe glomerular lesions were always accompanied by marked albuminuria. However, massive proteinuria may be present even in cases of low IDGLs.

d) Serum lipids: Positive correlations were found between IDGLs and total cholesterol, total lipids and neutral fat of the serum (Figs. 7, 8 and 9). The correlation of IDGLs with serum neutral fat was especially remarkable. Non-esterified fatty acids did not show any correlation with IDGLs. It appeared that the total serum cholesterol level was more closely related to the incidence of nodular glomerular lesions.

e) Thromboplastic activity: Between IDGLs and serum thromboplastic activity there was a relatively clear positive correlation (Fig. 10). In this connection, it seems important that nodular lesions are always associated with capillary thrombosis of the glomeruli.

f) Creatinine clearance: Although a negative correlation significant at $5 \%$ level was found between IDGLs and creatinine clearance, the scatter of individual values was relatively large, as demonstrated in Fig. 11. Severe glomerular lesions were associated with remarkably reduced glomerular blood flow, but low creatinine clearance did not necessarily mean advanced glomerular involvement. 


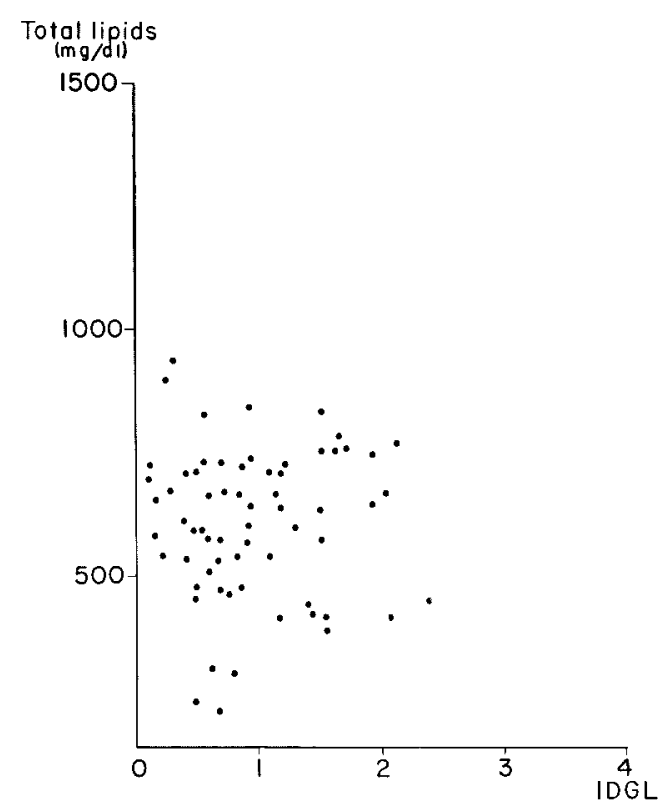

Fig. 7. IDGL against total plasmal lipids. A rough positive correlation is observed.

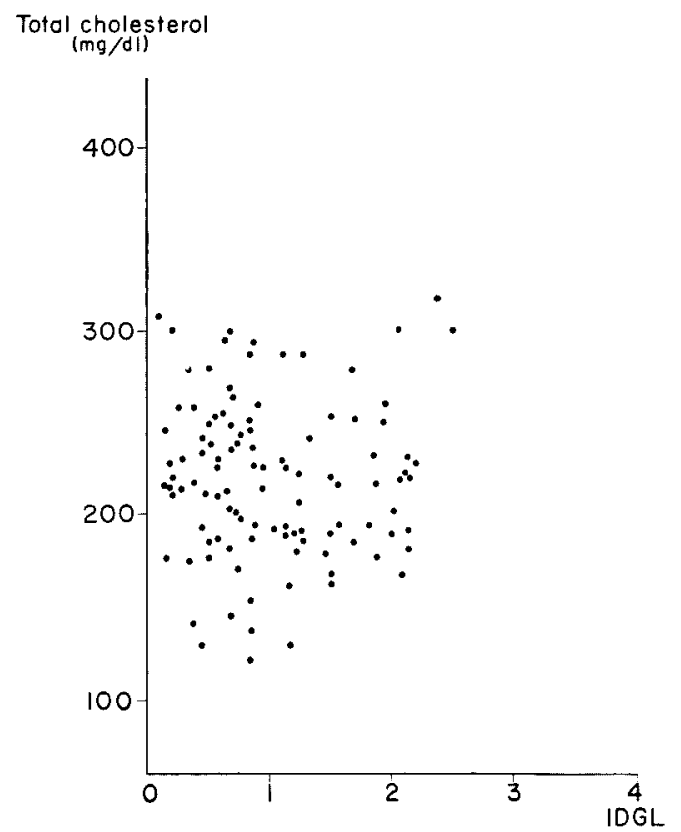

Fig. 8. IDGL against total plasmal cholesterol. A prsitive correlation is observed. 


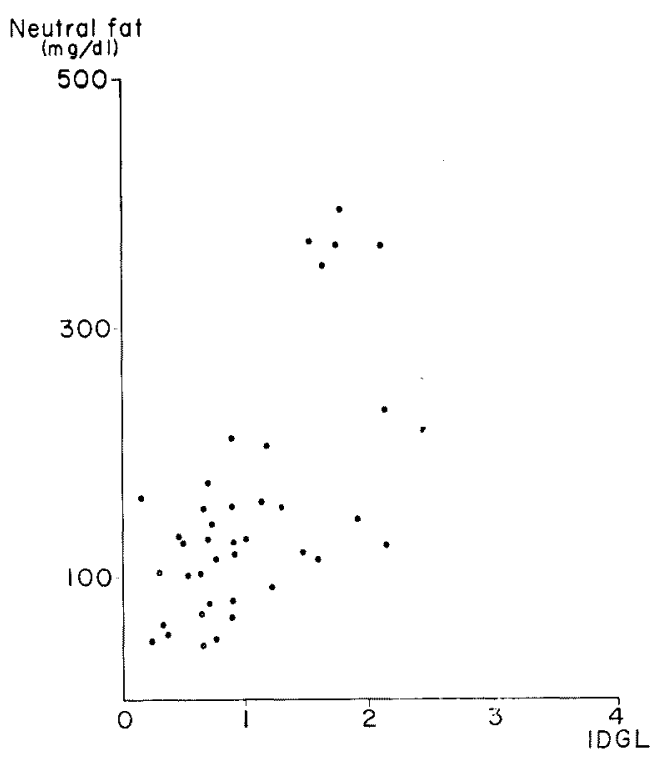

Fig. 9. A positive correlation is confirmed between IDGL and plasmal neutral fat. The correlation is more evident than in the case of cholesterol.

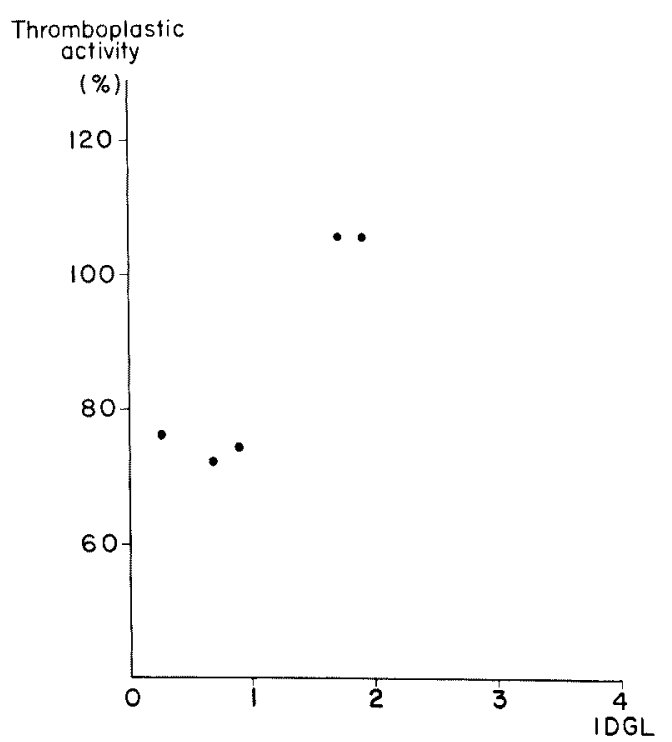

Fig. 10. A positive correlation is found between IDGL and plasmal thromboplastin activity. 


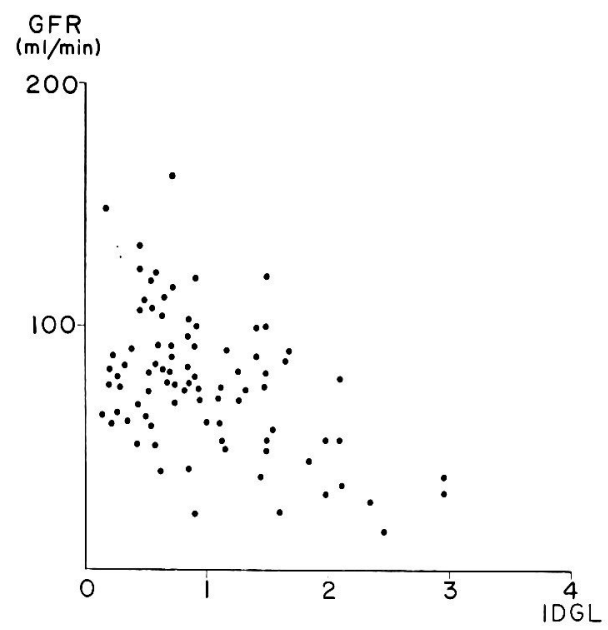

Fig. 11. A negative correlation is found between IDGL and GFR. The significance of the correlation depends mainly on the relation that severe glomerular involvement is always associated with distinctly lowered GFR. In the region of lower IDGL the correlation is obscured.

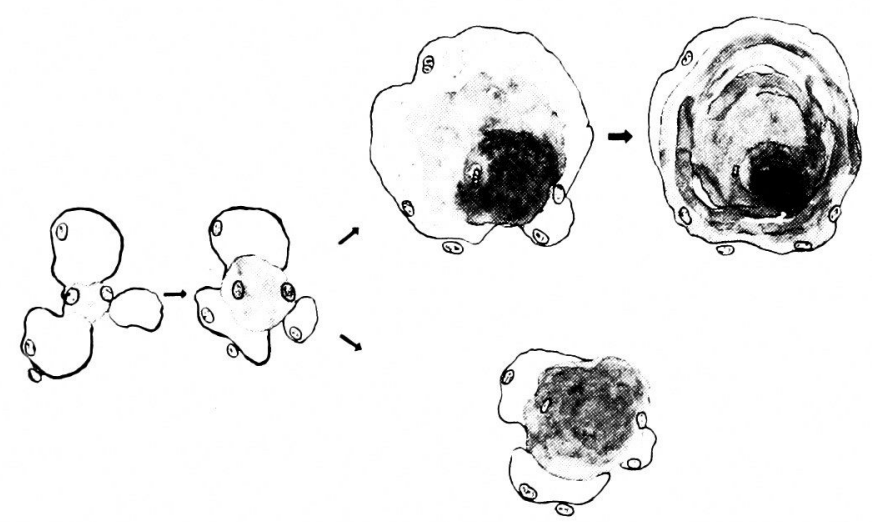

Fig. 12. The development of diabetic nodular glomerular lesions is illustrated. Simple hyaline deposits in the mesangial tissue can produce only small nodules, and larger nodules presuppose mesangiolytic changes. The mesangial tissue is swollen on account of plasmal infiltration, coalesced with plasmal precipitates and provides initial lesions of large nodules.

g) There were no distinct correlations of IDGLs with fasting blood sugar level, PAH clearance, non-protein nitrogen of the blood, and blood pressure.

In the above-mentioned results two points seem to be of special importance. One of them is that pronounced diabetic glomerular lesions develop only after a long duration of the disease. The other is that the glomerular involvement is associated with high serum lipids, most distinctly with high levels of serum neutral fat, but not with high blood sugar level. The effect of serum lipids on the develop- 
ment of diabetic glomerular lesions is also documented by histological findings. In thickened mesangial tissues fine fat droplets can occasionally be demonstrated. It is further a common observation that a large amount of fat droplets is demonstrated in intracapillary thrombotic mass, which is closely related to the development of nodular lesions.

\section{3) The histogenesis of nodular lesions}

On account of its pathognomonic features the nodular glomerular lesion has been a matter of concern of pathologists since the original report of Kimmelstiel and Wilson. In spite of its distinct histological character, the lesion was found in the present study only in connection with widespread diffuse lesions. There was not a single case in which the nodules were observed without extensive diffuse mesangial thickening even in glomeruli not involved in nodule formation. The finding leads inevitably to the conclusion, that nodular lesions can develop only on advanced diffuse lesions. However, the nodules are not a simple focal exaggeration of diffuse mesangial changes. Hyaline deposits in the mesangial tissue are of course irregular and there may be foci of accentuated hyaline deposition assuming the character of small nodules. But they never develop in this way to characteristic large nodules, which can be formed only on the basis of more drastic destruction of the capillary tufts.

In a series of biopsy specimens, a finding was obtained which offered a suggestion about the histogenesis of nodular lesions. In a part of glomerular capillary tufts the capillary basement membrane was detached from its mesangial junction and formed a wide blood space which was distinct from normal capillary lumina. The mesangial tissue was exposed to the space with a large contact surface and assumed edematous and swollen appearance. The normally intensively PAS and aniline blue positive constituents of the mesangial tissue were found to be split into fine fibrillar structures with blurred boundaries to the blood space. The finding indicated a rather severe destruction of the capillary structure and disintegration of the mesangial tissue, which might be most adequately called 'mesangiolysis'.

The swelling of the mesangial tissue was most probably caused by plasmal infiltration and the area attained already a size of the typical nodular lesion. It was easily conceivable that the plasma infiltrated lesion would turn to solid hyaline mass after a lapse of time especially when the plasma was rich in lipids. The lesion was often accompanied by fibrinous precipitates in the enlarged blood space occasionally with trapped and destroyed erythrocytes. The process could further culminate in complete fibrin thrombosis. The lesion of this type is usually called 'exudative', but in reality it has nothing to do with exudation. It represents merely intracapillary coagulation of blood plasma.

With the lapse of time coagulated plasma was partly ingested by endothelial phagocytes with abundant and foamy cytoplasm. The greater part of the mass, however, seemed to persist, become fused with adjoining mesangial tissues and 
finally to be transformed into coarse hyaline mass. It was noteworthy that proliferation of mesangial nuclei did not take place in the course of nodule formation in remarkable contrast with chronic glomerulonephritis.

Capillary thrombosis with or without mesangiolysis was an important pathological process in obliterating the glomeruli in the terminal stage of diabetic nephropathy and caused death due to uremia. IDGLs in such cases were very high and exceeded 3 , while the indices were much lower in death due to infections, most frequently acute suppurative pyelonephritis. Glomerular capillary thrombosis is especially apt to occur when hyaline thickening of the mesangia] tissue has attained a certain grade so as to encumber glomerular circulation. The process seems to contribute much to accelerating diabetic glomerular lesions in the terminal stage.

\section{References}

1) Kimmelstiel, P. \& Wilson, C. Intercapillary lesions in the glomeruli of the kidney. Amer. J. Path., 1936, 12, 83-98.

2) Bell, E.T. Renal lesions in diabetes mellitus. Amer. J. Path., 1942, 18, 744-745.

3) Laipply, T.C., Eitzen, O. \& Dutra, F.R. Intercapillary glomerulosclerosis. Arch. intern. Med., 1944, 74, 354-364.

4) Henderson, L.L., Sprau, R.C. \& Wagener, H.P. Intercapillary glomerulosclerosis. Amer. J. Med., 1947, 3, 131-144.

5) Bell, E.T. Renal Diseases. Lea \& Febiger, Philadelphia, 1950, pp. 402-405.

6) Allen, A.C. So-called intercapillary glomerulosclerosis. A lesion associated with diabetes mellitus. Arch. Path., 1941, 32, 33-51.

7) Fahr, T. Über Glomerulosklerose. Virchows Arch. path. Anat. 1942, 309, 16-33.

8) Farquhar, M.G., Hopper, J. \& Moon, H.D. Diabetic glomerulosclerosis. Electron and light microscopic studies. Amer. J. Path., 1959, 35, 721-753.

9) Kimmelstiel, P., Kim, O.J. \& Beres, J. Studies on renal biopsy specimens, with the aid of the electron microscope. 1. Glomeruli in diabetes. Amer. J. clin. Path., 1962, $38,270-279$.

10) Rogers, J., Robbins, S.L. \& Jeghers, H. Intereapillary glomeruloselerosis. A clinical and pathologic study. II. A clinical study of 100 anatomically proven cases. Amer. J. Med., 1952, 12, 692-699.

11) Brun, C., Gormsen, H., Hilden, T., Iversen, P. \& Raaschou, F. Diabetic nephropathy. Kidney biopsy and renal function tests. Amer. J. Med., 1953, 15, 187-197.

12) Daysog, A., Dobson, H.L. \& Brennan, J.C. Renal glomerular and vascular lesions in prediabetes and in diabetes mellitus. A study based on renal biopsies. Ann. intern. Med., 1961, 54, 672-684. 
Fig. 13. Typical diabetic lesion of the diffuse type is demonstrated. Biopsy specimen. Although there is nothing qualitatively pathognomonic in the histological picture, hyaline thickening of the mesangial tissue of this extent without proliferation of mesangial nuclei makes the diagnosis of diabetes practically possible, as documented by the result of histometrical determinations.

Fig. 14. Advanced diabetic glomerular lesion of the diffuse type with beginning nodule formation. The structure of glomerular capillaries is still preserved.

Fig. 15. Higher magnification of the left lower part of the glomerulus of Fig. 14. Small hyaline nodules are demonstrated. Such small nodules are regarded as locally accentuated hyaline thickening of the mesangial tissue, because the adjacent capillaries maintain their normal junctions to the mesangial tissue. 


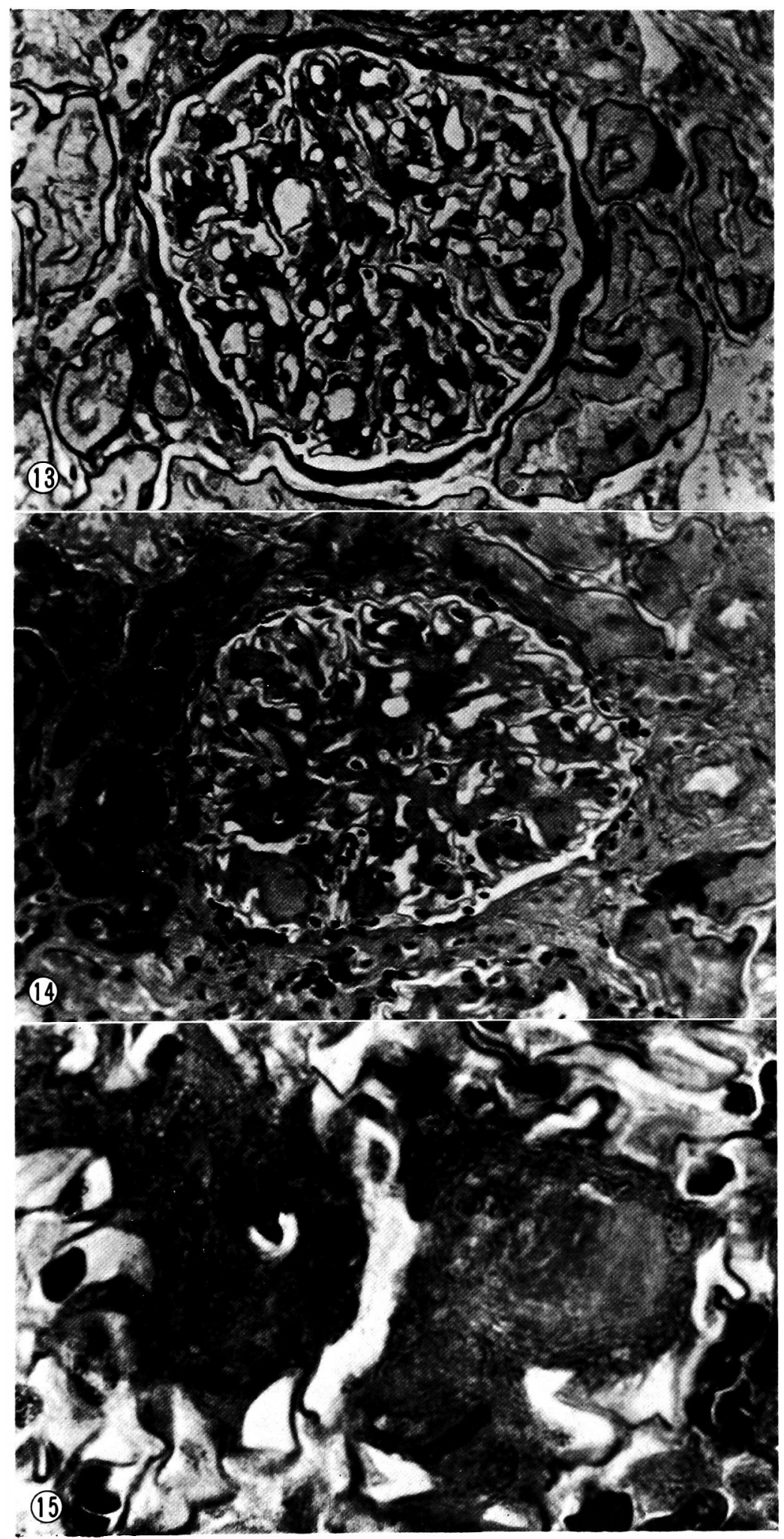


Fig. 16. Fresh mesangiolytic change of a capillary loop. The distended blood space contains fibrinous precipitates mixed with debris of erythrocytes. The development of such a large blood space is possible only when the capillary basement membrane is detached from its mesangial junctions. Compare normal capillary sections. The mesangial tissue protruding into the distended bloud space is swollen and edematous with obscured boundaries.

Fig. 17. Distended blcod space is filled with fine fibrillar extensions of the mesangial tissues. The picture is interpreted as representing a repair process ensuing on mesangiolytic changes as demonstrated in Fig. 16.

Fig. 18. Pre-existing mesangial tissue and capillary basement membrane are demonstrated intensively black by methenamine silver stain. Newly formed fibrillar structures filling the distended blood space are only partly positive for the stain. As complete nodules are usually in toto strongly positive for the stain, the picture of this figure is interpreted as representing an initial stage of the nodular lesion. 


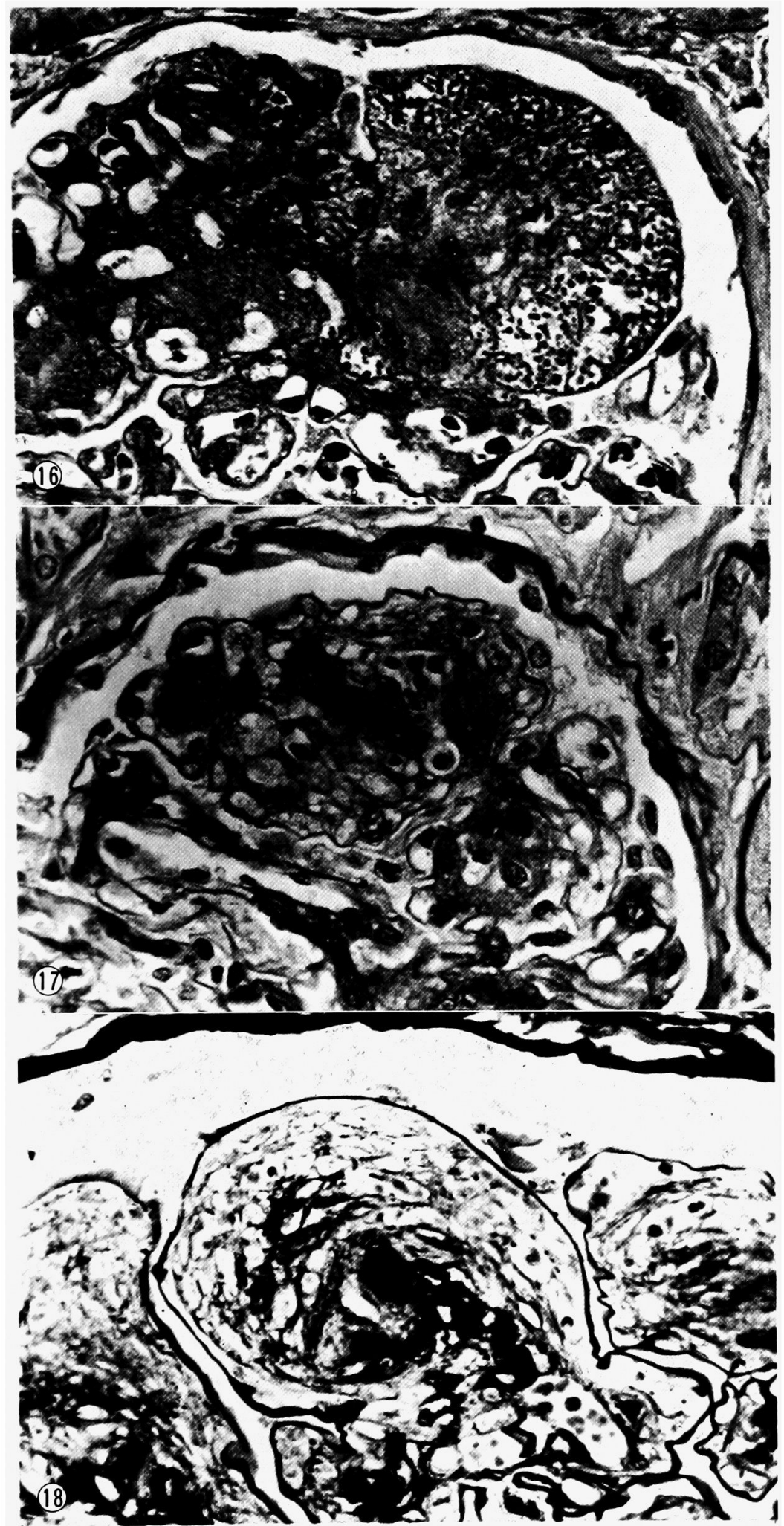


Fig. 19. Complete large nodular lesions of a diabetic kidney. The size of the nodules already excludes the possibility of their development through simple mesangial thickening.

Fig. 20. Intracapillary plasmal precipitates are partly ingested by phagocytes of endothelial origin. Note large foamy cytoplasm of the phagocytes, which is positive for fat stains and suggests high lipid contents of plasma.

Fig. 21. Thrombotic obliteration of glomerular capillaries. Fat stain. Note intensively positive stain which indicates high lipid contents of plasma. Fine fat droplets are also observed in glomerular epithelial cells and mesangial tissues. 


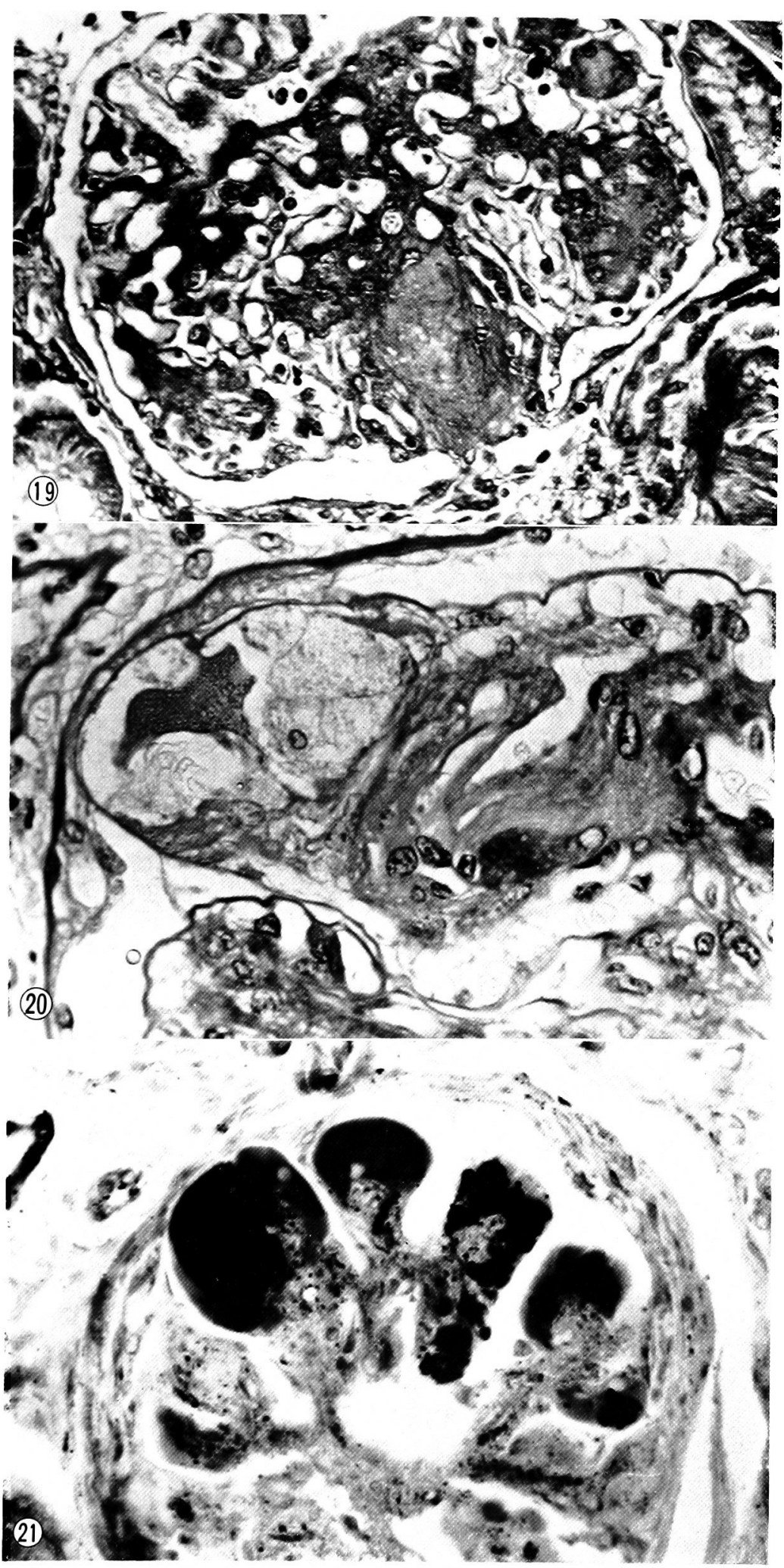

